\title{
Visual attention amplifies response priming of pointing movements to color targets
}

\author{
Thomas Schmidt And Anna Seydell \\ University of Giessen, Giessen, Germany
}

\begin{abstract}
We studied the influence of spatial visual attention on the time course of primed pointing movements. We measured pointing responses to color targets preceded by color stimuli priming either the same response as the target or the opposite response. The effects of visual attention at the prime and target locations were studied by varying both the cue-prime and prime-target intervals when presenting either exogenous attentional cues or, in a separate experiment, endogenous cues whose processing was a precondition for performing the task. Pointing trajectories revealed large priming effects in which pointing responses were first controlled by prime signals and then captured in midflight by target signals. Priming effects were strongly amplified when the relevant prime locations were visually attended at optimal cue-prime SOAs, with attention modulating the entire time course of the primed pointing movements. We propose that visual attention amplifies the earliest waves of visuomotor feedforward information, elicited in turn by primes and by targets.
\end{abstract}

\section{Visual Attention and Feedforward Processing}

The modulating effects of visual attention on cortical stimulus processing have been studied extensively. There are several mechanisms through which visual selective attention modulates the responses of individual cells (Kastner \& Ungerleider, 2000; Yantis \& Serences, 2003), one of which is the modulation of processing sensitivity (see, e.g., Carrasco, Penpeci-Talgar, \& Eckstein, 2000; Corbetta, Miezin, Dobmeyer, Shulman, \& Petersen, 1990). Treue and Martínez Trujillo (1999) showed that attention directed to a particular stimulus feature enhanced the response of cells in cortical area MT selective for that stimulus feature. This enhancement was multiplicative, affecting the entire tuning curve. Similar results have been reported for area V4 (McAdams \& Maunsell, 1999; Reynolds, Pasternak, \& Desimone, 2000), indicating that the effects of attentional enhancement are similar to those of an increase in effective stimulus contrast (e.g., Boynton, 2005; Reynolds \& Chelazzi, 2004).

Recently, theoreticians have started to distinguish between two fundamentally different types of visual processing: a rapid feedforward process, in which visual activation proceeds in a bottom-up direction through the visual system (Bullier, 2001; Thorpe, Fize, \& Marlot, 1996; VanRullen \& Koch, 2003; VanRullen \& Thorpe, 2002), and the slower, recurrent interactions developing in the immediate wake of this "fast feedforward sweep" (Lamme, 2000, 2006; Lamme \& Roelfsema, 2000). ${ }^{1}$ In the context of such models, visual attention is typically conceived of as the top-down influence of areas late in the processing stream on earlier areas via feedback connections, and is thereby linked to reentrant activity developing during pro- cessing of a stimulus, as well as the development of visual awareness (Di Lollo, Enns, \& Rensink, 2000; Lamme, 2000, 2006; Lamme \& Roelfsema, 2000; Lamme, Zipser, $\&$ Spekreijse, 2002; Tong, 2003).

However, reentrant attentional processes modulating the response properties of early visual areas could also play a part in the feedforward component of visual processing: If attentional selection is complete before the critical stimuli are presented, attention should be able to aid in all phases of the subsequent stimulus processing (Desimone \& Duncan, 1995). In this article, we are interested in such effects of attention on the bottom-up flow of information, which we have examined via a response-priming paradigm. We investigated the time course of primed pointing movements because this variant of response priming has been strongly linked to the initial feedforward component of visuomotor processing.

\section{Response Priming and Rapid-Chase Theory}

In the standard response-priming paradigm (Klotz \& Neumann, 1999; Neumann \& Klotz, 1994), participants have to perform a speeded response to a target stimulus that is preceded by a prime stimulus that triggers either the same response as the target (consistent prime) or the opposite response (inconsistent prime). Consistent primes speed responses, whereas inconsistent primes prolong them, and this priming effect increases with the stimulus onset asynchrony (SOA) between the prime and target, up to SOAs of about $100 \mathrm{msec}$ (Vorberg, Mattler, Heinecke, Schmidt, \& Schwarzbach, 2003). Inconsistent primes can actually elicit the wrong response, particularly at long SOAs, whereas response errors rarely occur with consis-

T. Schmidt, thomas.schmidt@psychol.uni-giessen.de 
tent primes. Strikingly, response-priming effects are independent of visual awareness of the prime: The increase in such effects with SOA is invariant, whether the prime can be identified perfectly or not at all and whether prime identification performance increases or decreases with SOA (Mattler, 2003; Schmidt \& Vorberg, 2006; Vorberg et al., 2003). These dissociations suggest that priming effects reflect a fast-occurring impact of the prime on motor responses that operates independently of subsequent masking processes, whereas visual awareness of the prime develops later and integrates information from both primes and masks (Di Lollo et al., 2000; Lamme et al., 2002).

Results from psychophysiological and imaging studies have indeed shown that primes directly initiate the specific motor responses assigned to them, leading to elicitation of both lateralized readiness potentials (LRPs; Eimer \& Schlaghecken, 1998, 2002, 2003; Leuthold \& Kopp, 1998; Vath \& Schmidt, 2007; Verleger, Jaśkowski, Aydemir, van der Lubbe, \& Groen, 2004) and lateralized metabolic activity in motor areas (Dehaene et al., 1998). These effects can also be traced in overt pointing responses. In a study by Schmidt, Niehaus, and Nagel (2006; Schmidt, 2002), one red and one green target were presented simultaneously in diagonally opposite quadrants of a display, preceded by one red and one green prime at the same two locations. Primes could have either the same colors as the targets (consistent primes) or colors that were switched with respect to the target colors (inconsistent primes). Speeded pointing responses toward the target of an appointed color were strongly affected by the primes: The onset of the pointing movements was timelocked to primes rather than to targets, and in many trials the initial response was actually in the direction of the primes, even if this movement direction turned out to be incorrect, so that the movement had to be reversed in midflight. Importantly, the early time course of the pointing trajectories was invariant across different target types and prime-target SOAs. This crucial finding suggests that the pointing responses initially were independent of all target characteristics, driven solely by the prime signal.

Schmidt et al. (2006; Vath \& Schmidt, 2007; see Vorberg et al., 2003, for a mathematical model) proposed a rapidchase theory of response priming that links visuomotor priming to feedforward processing. This framework assumes that the prime and target signals are transmitted sequentially by early feedforward waves of visuomotor processing (Bullier, 2001; Lamme \& Roelfsema, 2000; Thorpe et al., 1996; VanRullen \& Thorpe, 2002) elicited in turn by the primes and the targets. The prime and target signals directly initiate the motor responses assigned to them, with no need for conscious mediation (i.e., direct parameter specification; Neumann, 1990; Neumann \& Klotz, 1994; cf. Kunde, Kiesel, \& Hoffmann, 2003). The prime signal reaches executive motor areas first, initiating a response and continuing to drive that response on its own. After a delay about the size of the prime-target SOA, the target signal arrives and takes over response control from the prime signal. Priming effects, as well as the error rates in inconsistent trials, increase with prime-target SOA, because the prime has more time to drive the response on its own when the target is delayed longer. Response priming may occur overtly, with noticeable deflections of response trajectories in the direction of the prime, or covertly, only visible in the delayed onset of movements after response conflicts are resolved. ${ }^{2}$

In a simple feedforward system, the prime and target signals should traverse the system in strict successionthat is, the earliest signal arriving in executive motor areas should carry prime-related information exclusively, with no admixture of target information. In other words, visual signals entering the system in strict succession should generate strictly sequential motor output. The feedforward properties of such a system would show in the time course of the response: (1) Prime rather than target signals should determine the onset (and initial direction) of a response; (2) target signals should be able to influence the response before it is completed; and, crucially, (3) movement kinematics should initially depend on prime characteristics only and should be independent of all target characteristics (rapid-chase criteria; Schmidt et al., 2006). Note that the rapid-chase criteria do not guarantee that a stimulusresponse system is strictly feedforward in all its substages, and indeed they do not require such a strict assumption; they do reveal, however, when a system is behaviorally equivalent to a simple feedforward system. All three criteria were beautifully met by the data in Schmidt et al.'s study, as well as in comparable studies using pointing movements and LRPs (Schmidt, 2002; Schmidt \& Schmidt, 2008; Vath \& Schmidt, 2007), linking response priming to sequential feedforward processing of prime and target signals.

\section{Overview of the Experiments}

In this article, our goal is to show that visual attention deployed just in time before the presentation of the primes modulates the entire time course of the unfolding priming effect, in a manner consistent with modulation of the feedforward processing component - that is, in accordance with the rapid-chase criteria. In Experiment 1, modulation of the priming effect was induced by spatial precues supposed to summon attention exogenously toward or away from the relevant stimulus positions (Yantis \& Jonides, 1990). Experiment 2 extended this result to endogenous precues in a situation in which spatial selection was a necessary precondition for performing the task at all, so that all priming effects were conditional on selective attention (selection for action; Allport, 1989).

\section{EXPERIMENT 1}

We employed the primed-pointing procedure developed by Schmidt (2002; Schmidt et al., 2006). Two isoluminant annular targets, one red and one green, appeared in diagonally opposite quadrants of the display (Figure 1, upper row). Participants had to point from the center of the display to the target of appointed color. Just previous to the targets, two primes appeared, one red and one green, at the same two positions. The primes appeared for only $17 \mathrm{msec}$ at prime-target SOAs of 33,67 , or $100 \mathrm{msec}$. The primes and targets could correspond in color (consistent trials), or the prime colors could be switched with respect 
Cues

$17 \mathrm{msec}$

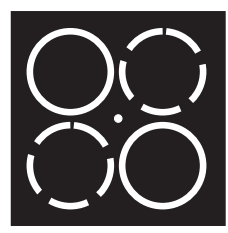

Cue

50 msec
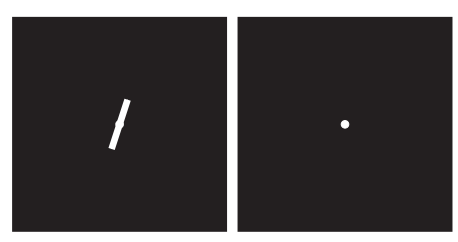

1

Cue-Prime SOA (cuing interval)

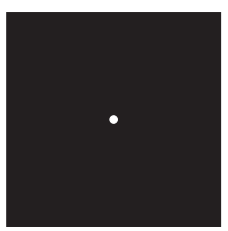

Primes

$17 \mathrm{msec}$

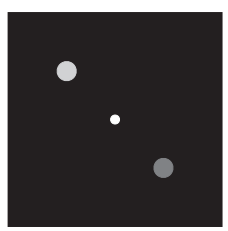

Prime-Target SOA
Primes

$17 \mathrm{msec}$
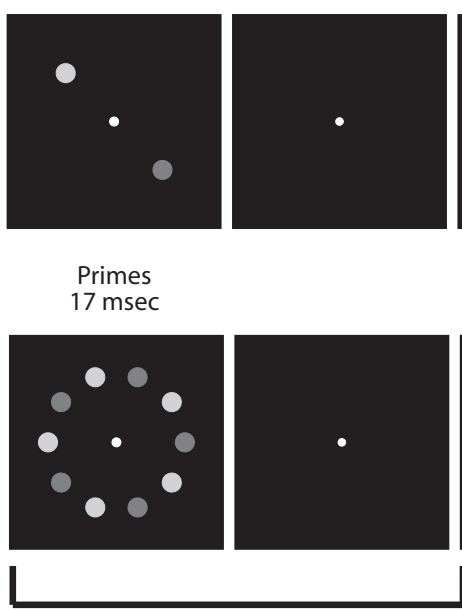

Targets

(until response)

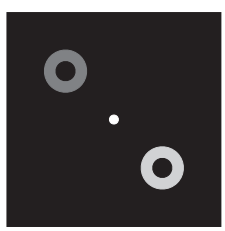

Targets

(until response)

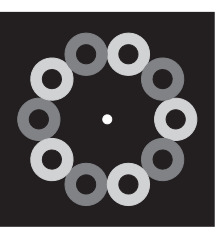

Exp. 2

Figure 1. Stimuli and tasks. In Experiment 1 (upper row), attentional cues were flashed either in the two quadrants in which primes and masks would appear (valid cues; here, the solid circles) or in the remaining quadrants (invalid cues; dashed circles). In Experiment 2 (lower row), 10 primes and 10 possible targets were presented, and the relevant locations of the primes and targets were indicated by a white bar. Both sequences depict inconsistent trials, in which primes and targets at corresponding positions have opposite colors. Light and dark gray indicate isoluminant red and green, respectively. See the text for further details.

to the target colors (inconsistent trials). Consistent and inconsistent primes were expected to initiate responses in the correct or in the opposite direction, respectively. The primes were efficiently masked by the subsequent targets and hardly visible; the participants were not informed that the primes were present (metacontrast; Breitmeyer \& Öğmen, 2006; Francis, 1997). Prime identification performance was not measured explicitly.

Spatially selective attention was manipulated via two exogenous cues that appeared simultaneously $100 \mathrm{msec}$ prior to prime onset (Figure 1, upper row). The cues were two annuli centered either on the positions where the primes and targets would appear (valid trials) or on the positions that would remain empty (invalid trials). The cues were valid in $50 \%$ of the trials and thus were unpredictive of stimulus locations or correct responses. We expected that the cues would automatically summon attention to either the stimulus or the nonstimulus locations, so that priming could be compared for attended and unattended stimuli.

\section{Method}

Participants. Ten right-handed students from the University of Göttingen (8 female, 2 male, ages 19-56) with normal or correctedto-normal vision participated for course credit or for a payment of $€ 24$. One additional participant had to be dismissed because he had difficulty distinguishing the red from the green targets. The experiment was preceded by a flicker photometry task to establish isoluminant colors. Each participant responded to only one color (either red or green, counterbalanced across participants) throughout all sessions. The participants were debriefed after the final session and received an explanation of the experiment. All gave informed consent and were treated in accordance with the ethical guidelines of the American Psychological Association.

Apparatus. The experiment was controlled by a $300-\mathrm{MHz}$ personal computer driving a 14 -in. VGA color monitor $(640 \times 480$ pixels) in synchrony with the monitor retrace rate of $60 \mathrm{~Hz}$. The monitor image was projected onto a workspace via a set of two mirrors, such that participants had the impression that the stimuli appeared directly on the workspace where they could interact with them. The workspace was tilted toward the participant at $44^{\circ}$ out of the transverse plane, at a viewing distance of $70 \mathrm{~cm}$. Pointing responses were recorded by a Polhemus FASTRAK magnetic tracking device at a sampling frequency of $120 \mathrm{~Hz}$. The sensor was referenced to the tip of a handheld stylus, and the workspace was illuminated so that participants could view the hand and stylus superimposed on the stimuli. Participants initiated each trial by placing the tip of the stylus on the fixation point with one hand and pressing the space bar with the other.

Stimuli. The primes were small disks (diameter $7.6 \mathrm{~mm} ; 1 \mathrm{~mm} \approx$ $0.082^{\circ}$ of visual angle) presented against a dark background $\left(0.07 \mathrm{~cd} / \mathrm{m}^{2}\right)$. The targets were annuli with an outer diameter of $15.2 \mathrm{~mm}$ and an inner diameter the size of the primes. An $83.00-\mathrm{cd} / \mathrm{m}^{2}$ fixation point (diameter $1.4 \mathrm{~mm}$ ) was in the center of the screen. Green stimuli were desaturated with CIE coordinates of $Y=11.50 \mathrm{~cd} / \mathrm{m}^{2}$, $x=.322$, and $y=.398$, whereas isoluminant red stimuli were matched to the green stimuli by heterochromatic flicker photometry for each participant. The stimulus conditions occurred equiprobably and pseudorandomly in a completely crossed repeated measures design.

Procedure. The fixation point appeared after participants had manually initiated the trial. Following a fixation period, attentional cues (diameter $19.0 \mathrm{~mm}, 83 \mathrm{~cd} / \mathrm{m}^{2}$ ) were presented in two diagonally opposite quadrants $53.7 \mathrm{~mm}$ from fixation (Figure 1, upper row). In the valid $50 \%$ of trials, these were the positions later occupied 
by the primes and targets; in the invalid $50 \%$ of trials, these were the two empty quadrants. After a cue-prime SOA of $100 \mathrm{msec}$, one red and one green prime were presented for $17 \mathrm{msec}$ in diagonally opposite quadrants of the display. After a prime-target SOA of 33, 67 , or $100 \mathrm{msec}$, one red and one green target were presented at the same locations as the primes, such that their colors were either consistent or inconsistent (switched) with respect to the prime colors. The participants were instructed to point as quickly as possible to the target of the appointed color. The period from fixation onset to target onset was fixed at $1,000 \mathrm{msec}$. The targets remained onscreen until participants had finished a speeded pointing response toward either target. The participants took part in four experimental sessions, each consisting of one practice block followed by 30 blocks of 24 trials.

Statistical methods. A single session was missing because the participant had been unavailable, and practice blocks were excluded. Trials were excluded if a participant had hit one of the empty target locations or if the arrival time was shorter than $100 \mathrm{msec}$ or longer than $999 \mathrm{msec}$. This procedure eliminated $0.92 \%$ of all trials. ANOVAs are reported with Greenhouse-Geisser corrected $p$ values. Generally, only effects meeting a significance criterion of $p<.05$ are reported.

We analyzed the entire time course of the pointing trajectories, looking at the spatial position of the stylus sensor as a function of time. Pointing trajectories were simplified by projecting the horizontal and vertical coordinates of the stylus tip onto a line connecting the red and green targets, counting the starting point as zero and the correct target direction as positive. We established the trajectorial priming function as a spatiotemporal measure of priming by subtracting trajectories in consistent trials from those in inconsistent trials (negative values indicating how far the sensor position in inconsistent trials lagged behind the sensor position in consistent trials at corresponding points in time). Different aspects of priming could be evaluated by deriving kinematic parameters of the trajectories and the trajectorial priming functions. As a result, we could look at priming effects evaluated at different stages throughout the response, at priming effects in velocity profiles, and at various other statistics.

Kinematic parameters were extracted by jackknifing methods (Ulrich \& Miller, 2001). The pointing trajectories from the $n$ participants were averaged across subsamples of $(n-1)$ participants, excluding a different participant from each subsample. Movement parameters (e.g., arrival times and peak velocities) were estimated from each subsample and then averaged, allowing for estimates much more reliable than those from single participants. Because each participant's data enter each subsample but one, it can be shown that the variances and standard errors of the movement parameters extracted in this way are too small by factors of $(n-1)^{2}$ and $(n-1)$, respectively (Ulrich \& Miller, 2001). $F$ tests and standard error bars were corrected accordingly, in order to estimate variation among participants rather than subsamples.

\section{Results}

The upper panels of Figure 2 show how the primes affected the trajectories of pointing responses toward the correct target. After the primes and targets had occurred, the sensor remained at rest for a while. Roughly $200 \mathrm{msec}$ after prime onset, it started to move, and on average the movement direction was determined by the primes rather than the targets. In consistent trials, this was the correct direction, and the sensor traveled continuously in the direction of the correct target until the response was completed. In inconsistent trials, however, the sensor tended to detour into the quadrant specified by the misleading prime before reversing and proceeding in the correct direction. This detour was longer and reached farther into the wrong quadrant the more time elapsed between the primes and targets.

Priming effects early in the trajectory. We evaluated the priming effect at two points in the unfolding movement:
Shortly after movement onset, and shortly before arrival at the correct target location. Priming early in the trajectory was evaluated at a time (from prime onset) when the sensor had first moved at least $3 \mathrm{~mm}$ in the positive direction. ${ }^{3}$ This criterion threshold was crossed earlier in consistent than in inconsistent trials (at times $t_{\text {con }}$ and $t_{\text {incon }}$, respectively) $[F(1,9)=139.36, p<.001]$, and this priming effect $\left(t_{\text {incon }}-t_{\text {con }}\right)$ increased strongly with prime-target SOA $[F(2,18)=43.08, p<.001]$, reaching values of $150 \mathrm{msec}$ and more (Figure $3 \mathrm{~A}$ ). It is obvious from Figure 2 that these effects mainly reflected the time cost incurred by detours toward the misleading prime. Crossing times increased with SOA $[F(2,18)=22.13, p<.001]$, mainly because of increasing crossing times in inconsistent trials. Importantly, the priming effects were larger for valid than for invalid cues $[F(1,9)=8.93, p=.015]$.

Priming effects in arrival times. Arrival times (Figure $3 \mathrm{~B}$ ) were defined as times at which the sensor first entered a $17-\mathrm{mm}$ radius around the correct target position. They showed the same overall pattern as the priming effects evaluated at the earlier criterion, with earlier arrivals in consistent than in inconsistent trials $[F(1,9)=$ $121.61, p<.001]$ and a strong increase of priming with SOA $[F(2,18)=165.74, p<.001]$. Again, arrival times increased with $\operatorname{SOA}[F(2,18)=161.80, p<.001]$, mainly because of the increase in priming for inconsistent trials. Valid cues led to stronger priming effects than did invalid cues $[F(2,18)=37.44, p<.001]$, with additional interactions between validity, consistency, and SOA $[F(2,18)=$ $6.41, p=.012]$ and between validity and SOA $[F(2,18)=$ $6.15, p=.017]$, both indicating slight deviations from additivity. Response errors (i.e., arrivals at the wrong target location; Figure 3C) occurred almost exclusively in inconsistent trials, and error rates in inconsistent trials increased with SOA more steeply in valid than in invalid trials.

Priming effects in peak velocities. Overall, priming effects at arrival were markedly smaller than those evaluated at the earlier criterion. This happened because pointing movements in inconsistent trials became faster than those in consistent trials, allowing the inconsistent trajectories to partly catch up with the consistent ones (Figure 3D). Once the movements in inconsistent trials had completed their detour toward the misleading prime, they reached higher peak velocities than those in consistent trials $[F(1,9)=13.68, p=.005]$, and this difference became larger with SOA $[F(2,18)=7.82, p=.004]$. Overall, peak velocity increased with $\operatorname{SOA}[F(2,18)=4.56, p=.025]$.

Trajectorial priming effects. Trajectorial priming effects (Figure 2, lower panels) strongly depended on cue validity and SOA. Onset times of trajectorial priming effects were defined as the times (from prime onset) when the effect first fell below $-3 \mathrm{~mm}$ (i.e., when the sensor position in inconsistent trials lagged at least $3 \mathrm{~mm}$ behind that in consistent trials). With increasing SOA, these onset times became shorter $[F(2,18)=3.35, p=.06$; Figure 4A], peak amplitudes of the priming function became more negative $[F(2,18)=90.13, p<.001$; Figure 4B], and peak velocities of the priming function increased $[F(2,18)=31.94, p<.001$; Figure $4 \mathrm{C}]$. With valid as compared with invalid cues, peak amplitudes were more 


\section{Experiment 1: Pointing Trajectories}

Invalid
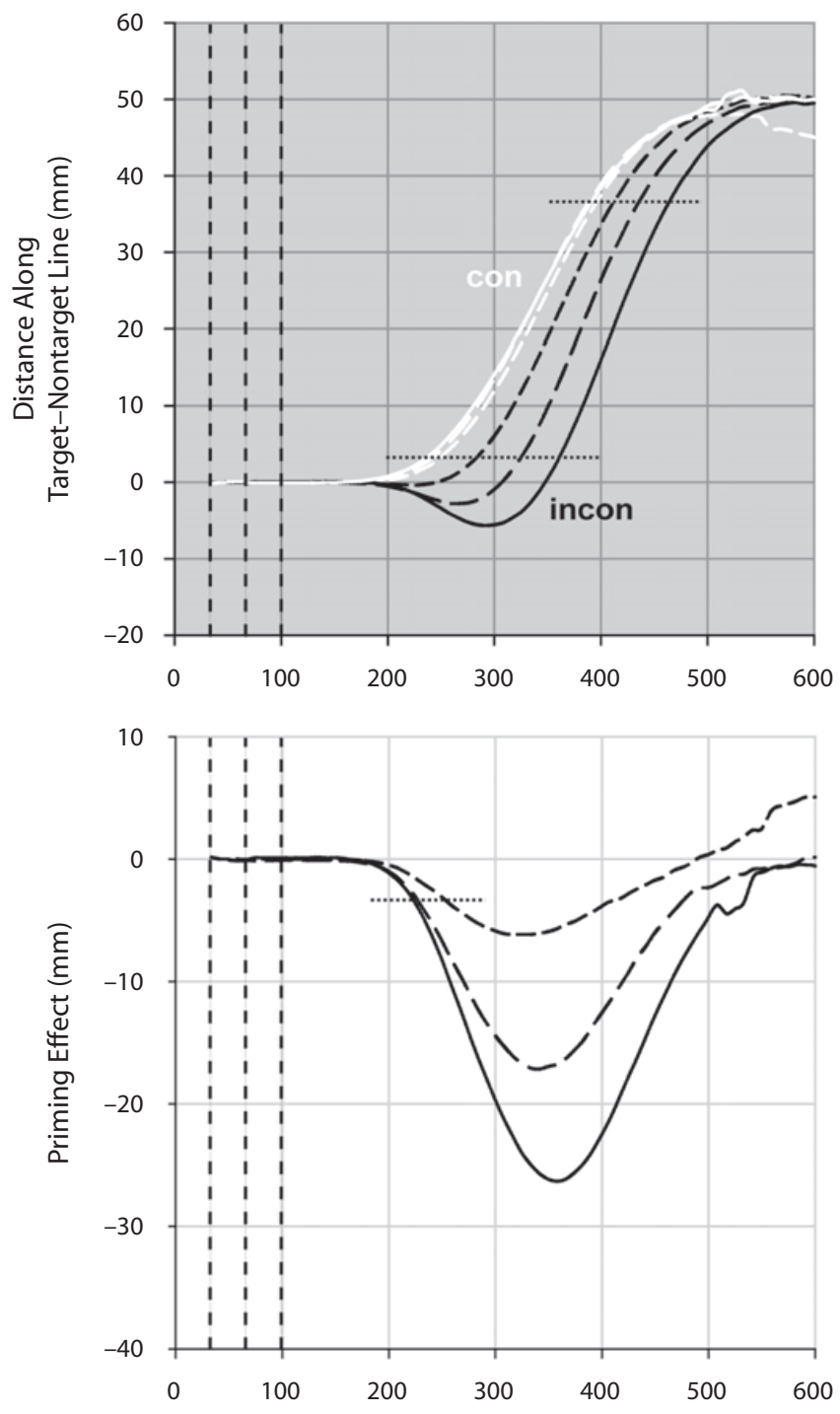

Valid
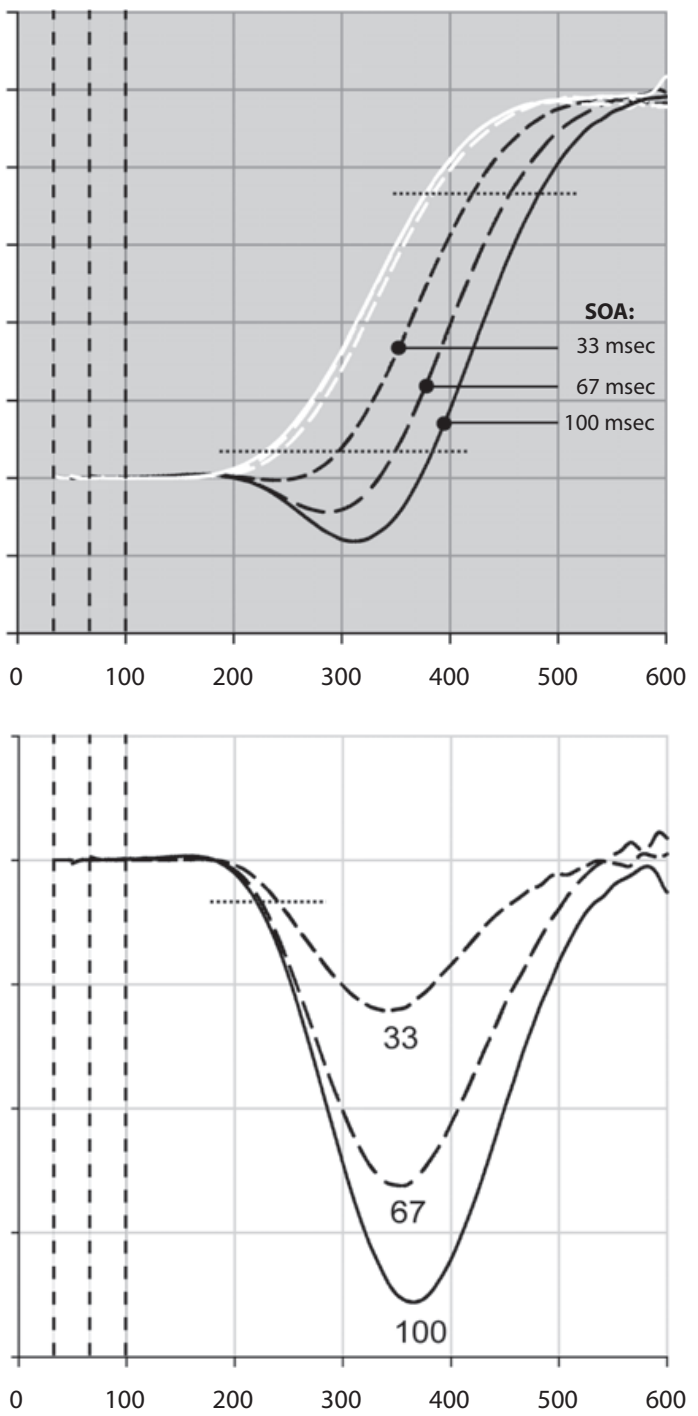

Time From Prime Onset (msec)

Figure 2. Upper panels: Pointing trajectories in Experiment 1. The dependent variable is the position of the sensor projected onto the target-nontarget line; zero denotes the fixation point, and positive values denote the correct target direction. Lower panels: Trajectorial priming functions obtained by subtracting consistent from inconsistent trials. Negative values indicate that the sensor position in inconsistent trials lags behind that in consistent trials. Throughout the figure, time axes are locked to prime onset, vertical dashed lines indicate the possible target onset times, and horizontal stippled lines indicate the various evaluation criteria.

negative $[F(1,9)=35.34, p<.001]$, and priming developed at higher velocity $[F(1,9)=14.30, p=.004]$.

\section{Discussion}

Our results are in line with our previous findings (Schmidt, 2002; Schmidt et al., 2006). On average, responses started at a fixed time following prime onset and initially went in the direction specified by the primes rather than the targets. When primes and targets were consistent, this initial direction was correct, and the sensor simply traveled toward the correct target until the response was completed. When primes and targets were inconsistent, however, the sensor initially traveled into the quadrant occupied by the misleading prime. This detour into the wrong quadrant lasted for a time directly dependent on the prime-target SOA; then, a movement would reverse and finally proceed in the correct direction. As a result, inconsistent primes delayed responses in the correct direction, and these delays increased with prime-target SOA. On average, pointing and priming onsets were strictly time 


\section{Experiment 1: Parameters of Pointing Trajectories}

A

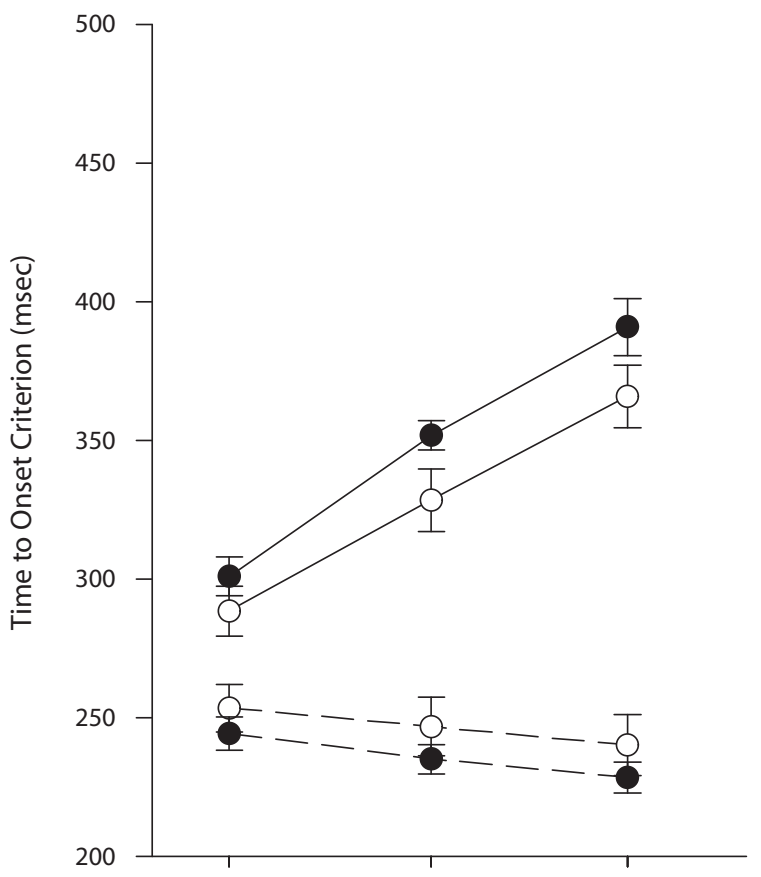

C

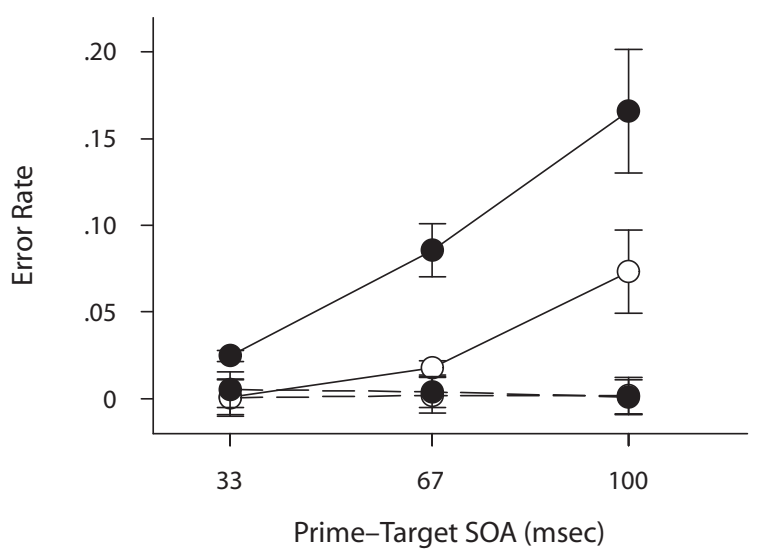

B

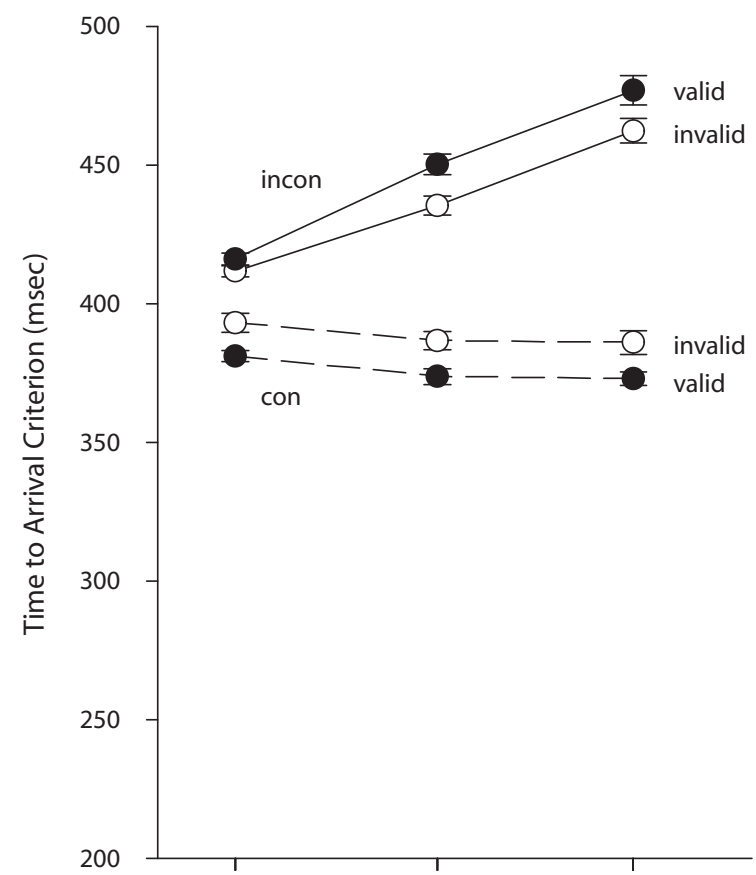

D

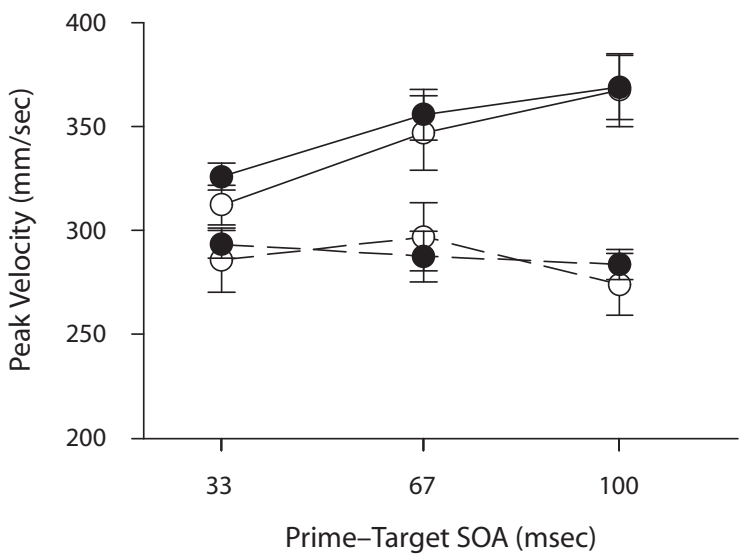

Figure 3. Parameters of the pointing trajectories: Time to an early criterion (A), time to arrival (B), error rate (C), and peak velocity (D). The early criterion was located on a radius $3 \mathrm{~mm}$ from fixation, in the direction of the correct target. The arrival criterion was located on a radius $17 \mathrm{~mm}$ around the correct target. All times are from prime onset. Here and in all subsequent figures, error bars denote the standard errors of the means estimated from jackknifed data (Ulrich \& Miller, 2001), with a correction for between-subjects variance (Loftus \& Masson, 1994).

locked with prime onset, even though participants were supposed to respond to the targets, whereas correction movements during inconsistent trials were time locked with target onset.

Priming effects were clearly affected by cue validity, suggesting modulation by visual attention. Relative to invalid cues, valid exogenous cues amplified priming effects early in the trajectory, late in the trajectory, and in the frequency of response errors. Attention modulated the entire time course of the pointing trajectory: The trajectorial priming function, defined as the spatial difference between consistent and inconsistent trajectories, reached larger peak amplitudes and higher peak velocities in valid than in invalid trials.

The overall data pattern suggests that pointing responses were controlled sequentially by prime and target signals, such that the response was initiated by the prime and captured in midflight by the target signal, as suggested by 


\section{Experiment 1: Parameters of Trajectorial Priming Functions}
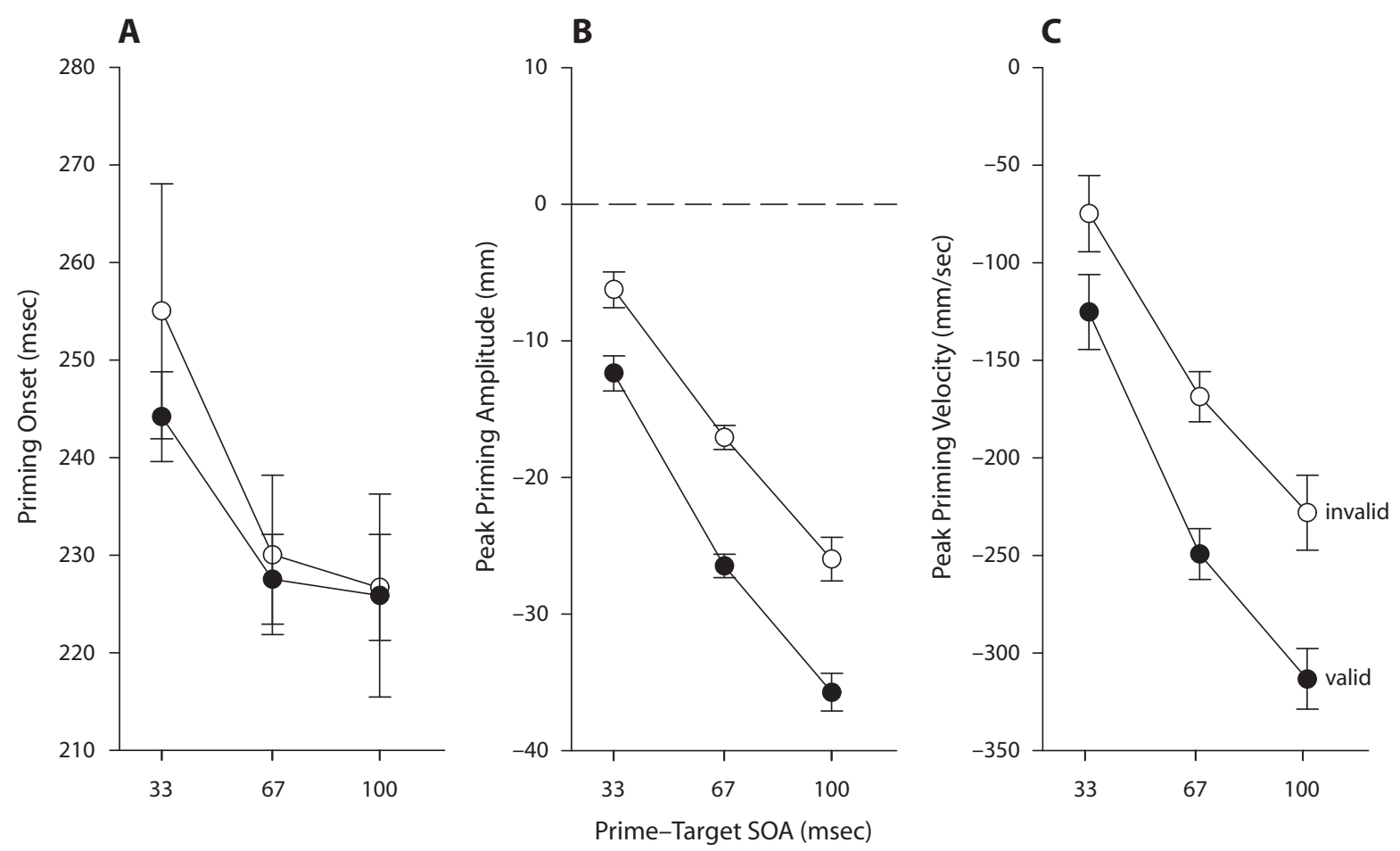

Figure 4. Parameters of trajectorial priming functions: Time to priming onset (A), peak amplitude of the priming function (B), and peak velocity of the priming function (C). Priming onset is defined as the point in time at which a priming function first falls below $-3 \mathbf{m m}$. All times are from prime onset.

rapid-chase theory (Schmidt et al., 2006; Vath \& Schmidt, 2007). This conclusion is corroborated by the analysis of error rates (i.e., actual arrivals at the incorrect target), which were low in consistent trials, when both prime and target activated the correct response, but higher in inconsistent trials, when the prime first activated a response in the incorrect direction. Error rates strongly increased with SOA in those trials, presumably reflecting responses that were driven by the misleading prime signal and that were not reversed by the target signal before they reached completion.

When inconsistently primed movements finally started to go in the correct direction, they became about $30 \%$ faster than consistently primed movements. This pattern had already been observed in earlier studies of primed pointing movements (Schmidt, 2002; Schmidt et al., 2006) and primed LRPs (Vath \& Schmidt, 2007). We can offer two explanations. First, the speedup for inconsistent trajectories might be related to the reversal of response-priming effects observed at SOAs beyond $100 \mathrm{msec}$ (Eimer \& Schlaghecken, 1998), whereby inconsistent primes lead to faster responses than do consistent ones. Although the source of this reversal is debated (Lleras \& Enns, 2004; Verleger et al., 2004), one explanation is that the prime-initiated response undergoes active self-inhibition (Schlaghecken \& Eimer, 2002). Trajectories in inconsistent trials might thus benefit from the earliest effects of self-inhibition of the prime-initiated incorrect response, which in turn might disinhibit the correct response. Second, Vorberg et al.'s (2003) accumulator model of response priming posits two accumulators collecting stochastic sensory evidence for the correct and incorrect responses, respectively. The two accumulators inhibit each other, implementing a winnertakes-all system. In the model, information accumulation in the correct direction occurs later but proceeds faster after an inconsistent prime has driven the process in the wrong direction, even without active self-inhibition.

\section{EXPERIMENT 2}

In Experiment 1, we used attention to modulate the dynamics of a visuomotor task that could have been completed without attentional cues. In Experiment 2, our goal was to create a task in which visual selective attention was a necessary precondition for performing the task at all, and also necessary for allowing a priming effect to develop.

We studied the impact of endogenously controlled attention (Yantis \& Serences, 2003) on priming by presenting a circular configuration of 10 possible targets preceded by 10 primes at the same positions (Figure 1, lower row). The stimulus configurations were such that neighboring stimuli would be alternatingly red and green, and a red target or prime would always lie opposite a green one. Participants responded to one pair of opposite targets by pointing to the target of the appointed color. To know 
Cue-Prime SOA: $100 \mathrm{msec}$
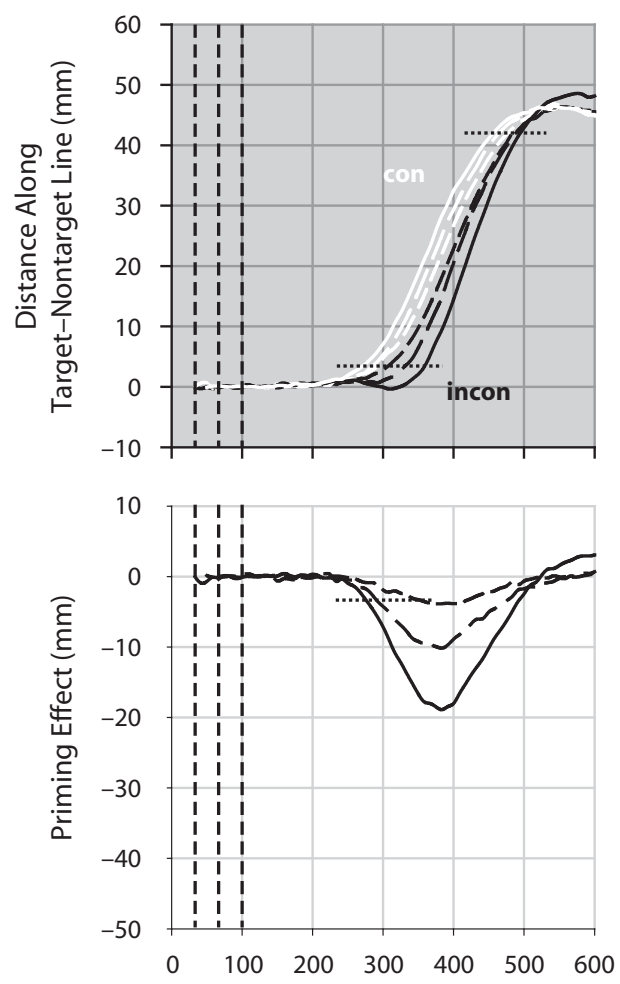

\section{Experiment 2: Pointing Trajectories}
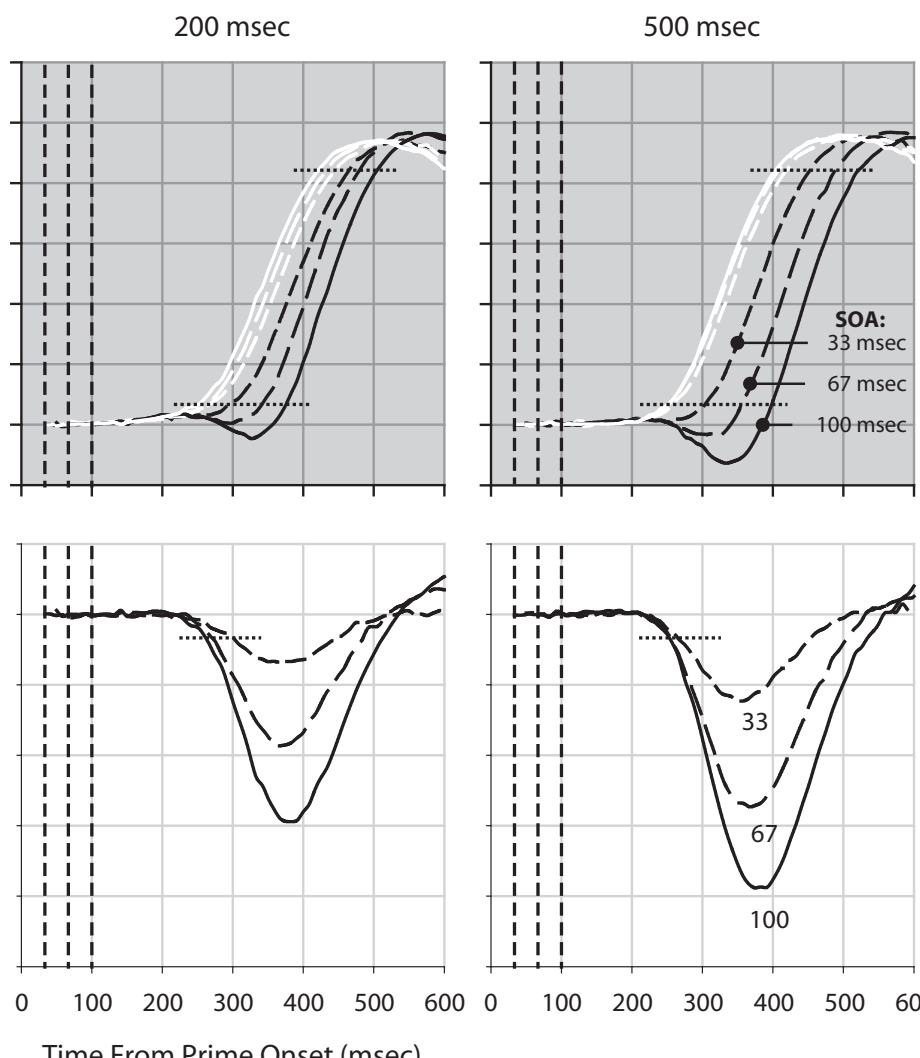

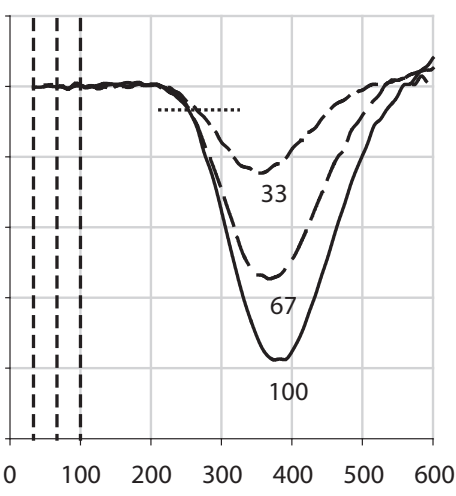

Time From Prime Onset (msec)

Figure 5. Trajectories (upper panels) and trajectorial priming functions (lower panels) in Experiment 2. The details are as in Figure 2.

which of the target pairs to respond to, they had to process a cue presented before prime onset that indicated the relevant pair of opposing targets. The twist of this design is that primes as well as targets should have a spatially neutral impact on pointing movements, unless spatial selection of the cued locations has taken place. Visual selection is thus necessary for performing the task (i.e., selection for action; Allport, 1989) and for obtaining spatially directed priming effects, not just a modulating influence on performance.

\section{Method}

Participants. Eight right-handed students from the University of Göttingen ( 7 female, 1 male, ages 19-25) with normal or corrected-tonormal vision participated for course credit or for a payment of $€ 27$.

Procedure. The apparatus and statistical methods were the same as in Experiment 1. The prime and target stimuli were also the same as in Experiment 1, but they appeared in a new arrangement (Figure 1 , lower row). The 10 possible targets were arranged in a circle $114.0 \mathrm{~mm}$ in diameter, such that neighboring targets would be alternately red and green, and a red target would always lie opposite a green one. Similarly, 10 colored primes were arranged at the same positions and with their colors either consistent or inconsistent (switched) with respect to the target colors. The cue was a white bar $\left(83.0 \mathrm{~cd} / \mathrm{m}^{2}\right)$ indicating one pair of opposing targets and extending from the fixation point by $17.8 \mathrm{~mm}$ in both directions.
After a fixation period, the cue was presented for $50 \mathrm{msec}$. At a cue-prime SOA (cuing interval) of 100, 200, or $500 \mathrm{msec}$, primes appeared for $17 \mathrm{msec}$. At a prime-target SOA of 33, 67, or $100 \mathrm{msec}$, the targets appeared and remained on screen until participants had finished a speeded pointing response toward the cued target of the appointed color or toward an incorrect target. The period from fixation onset to target onset was fixed at $1,000 \mathrm{msec}$. Participants took part in four experimental sessions, each consisting of 1 practice block followed by 20 blocks of 36 trials. The outlier detection procedure described for Experiment 1 eliminated 1.25\% of trials.

\section{Results}

Pointing trajectories (Figure 5, upper panels) were highly similar to those in Experiment 1. On average, the sensor initially started in the direction of the prime, and in inconsistent trials it continued to detour into the wrong quadrant for a time depending on the prime-target SOA. The effect increased with the duration of the cuing period and the prime-target SOA.

Priming effects early in the trajectory. As in Experiment 1 , the early criterion ( $3 \mathrm{~mm}$ in a positive direction) was crossed earlier in consistent than in inconsistent trials $[F(1,7)=81.95, p<.001]$, and this priming effect increased with prime-target SOA $[F(2,14)=31.25, p<$ .001 ; Figure $6 \mathrm{~A}]$. Crossing times generally increased with 
$\operatorname{SOA}[F(2,14)=27.92, p=.001]$, mainly due to increasing times in inconsistent trials. Importantly, priming effects also increased with cuing interval $[F(1,7)=6.47$, $p=.010]$, suggesting that primes had a larger impact on the response when more time was available to select the relevant positions.

Priming effects in arrival times. Arrival times (Figure $6 \mathrm{~B}$ ) showed the same overall pattern, with earlier arrivals in consistent than in inconsistent trials $[F(1,7)=$ 87.23, $p<.001]$ and a strong increase of this priming effect with SOA $[F(2,14)=55.23, p<.001]$. On average, arrival time increased with $\mathrm{SOA}[F(2,14)=30.08$, $p<.001]$, mainly because of the increase during inconsistent trials. Notably, priming effects also increased with cuing interval $[F(2,14)=35.74, p<.001]$, as did arrival times in general $[F(2,14)=8.97, p=.010]$. The increase in priming with prime-target SOA was steeper at longer cuing intervals, as confirmed by significant interactions between validity, consistency, and $\operatorname{SOA}[F(4,28)=7.14$, $p=.002]$ and between validity and SOA $[F(4,28)=15.27$, $p<.001]$. Again, response errors (Figure 6C) occurred almost exclusively in inconsistent trials and increased markedly with SOA and cuing interval.

Priming effects in peak velocities. In contrast to Experiment 1 , there was no significant effect of prime-target consistency on peak velocities $[F(1,7)=2.09, p=.192$; Figure 6D]. The only noteworthy effects were an increase in peak velocity with cuing interval $[F(2,14)=4.46, p=$ $.032]$ and prime-target SOA $[F(2,14)=3.23, p=.070]$.

Trajectorial priming effects. Trajectorial priming functions (Figure 5, lower panels) were established as in Experiment 1. Again, their time course strongly depended on the cuing interval and prime--target SOA. With increasing SOA, onset times became shorter $[F(2,14)=22.32$, $p<.001$; Figure 7A], peak amplitudes of the priming function became more negative $[F(2,14)=57.19, p<.001$; Figure $7 \mathrm{~B}]$, and the peak velocities of the priming function became higher $[F(2,14)=57.62, p<.001$; Figure $7 \mathrm{C}]$. Increasing cuing interval had a similar effect on the onset times, peak amplitudes, and peak velocities of the trajectorial priming effects $[F(2,14)=5.26,29.74$, and 8.57, respectively, all $p s \leq .020]$. The interaction between cuing interval and prime-target SOA was significant only for the peak amplitudes $[F(4,28)=7.14, p<.001]$, which increased more steeply with SOA at longer cuing intervals.

\section{Discussion}

Whereas Experiment 1 only demonstrated modulation of a priming effect by visual attention, Experiment 2 employed a selection-for-action paradigm in which spatial selection of the relevant locations was a prerequisite for performing the task at all. Priming effects were highly similar to those measured in Experiment 1 and in earlier studies (Schmidt, 2002; Schmidt et al., 2006) and clearly increased with cuing interval, suggesting that primes had a higher impact on responses to the targets when more time was available to select the relevant locations. Importantly, the priming effects were directional, leading away from the correct target position in inconsistent trials. These detours demonstrate that spatial selection of specific primes must have taken place, because without such selection, primes could not have exerted a spatially directed influence on the pointing response. Even though clear priming effects were observed in arrival times and error rates at a cuing interval as short as $100 \mathrm{msec}$, these effects nearly tripled in size when the cuing interval was increased to $500 \mathrm{msec}$. Again, attention modulated the entire time course of the pointing trajectory: The trajectorial priming function had an earlier onset, reached larger peak amplitudes, and developed with higher peak velocities, the longer that the cuing interval became.

These results suggest that with increasing cuing intervals, spatially selective attention molds itself more and more efficiently into the cued locations (McMains \& Somers, 2004). The earlier that attention is deployed at selected locations, the more it will enhance visuomotor processing of upcoming stimuli, thereby amplifying the effects of the primes. In contrast, if selection is still incomplete upon arrival of the primes, the priming effects will be reduced, because the prime display cannot initiate a spatially directed motor response.

\section{GENERAL DISCUSSION}

Both experiments reported here show that visual attention enhances response-priming effects generated by subsequently appearing stimuli. This enhancement encompasses all phases of primed pointing responses: With attention in place, responses start earlier, reach larger peak amplitudes, and can travel with higher peak velocities. In addition, the impact of attention can be more than just modulatory: Experiment 2 was designed in such a way that spatial selection was necessary not only for performing a spatially directed response, but also for obtaining a spatially directed priming effect.

Does attentional modulation affect the feedforward component of the pointing task? That is, in behavioral terms, are the priming effects consistent with Schmidt et al.'s (2006) rapid-chase criteria? Regarding only the later prime-target SOAs, the first two of these criteria are met in both experiments: Primes rather than targets determine the onset time and initial direction of the response (Criterion 1), and the target signal is able to redirect the response in midflight (Criterion 2). This data pattern suggests that at these SOAs responses are controlled sequentially by prime and target signals, but it does not exclude the possibility that these signals could mix or overlap prior to the level of response control. The third criterion therefore evaluates whether primes and targets might control the response in strict sequence, with no overlap between the signals, so that the initial phase of the priming effect is controlled by a signal containing only prime and no target information. This criterion requires that at each SOA, the trajectorial priming function must display an invariant early time course - in other words, all curves must initially lie on top of each other, only branching off after they have followed the exact same time course for a time that depends on the SOA. This criterion is met for the two later prime-target SOAs in all cuing conditions reported here, which does support rapid-chase processing. 


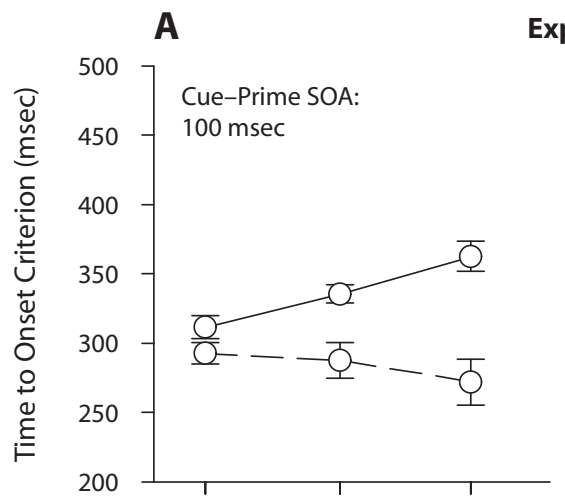

Experiment 2: Parameters of Pointing Trajectories

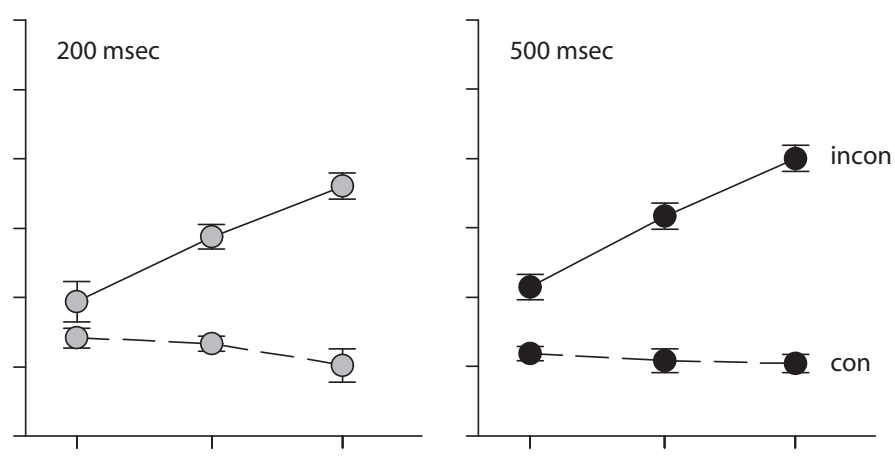

B
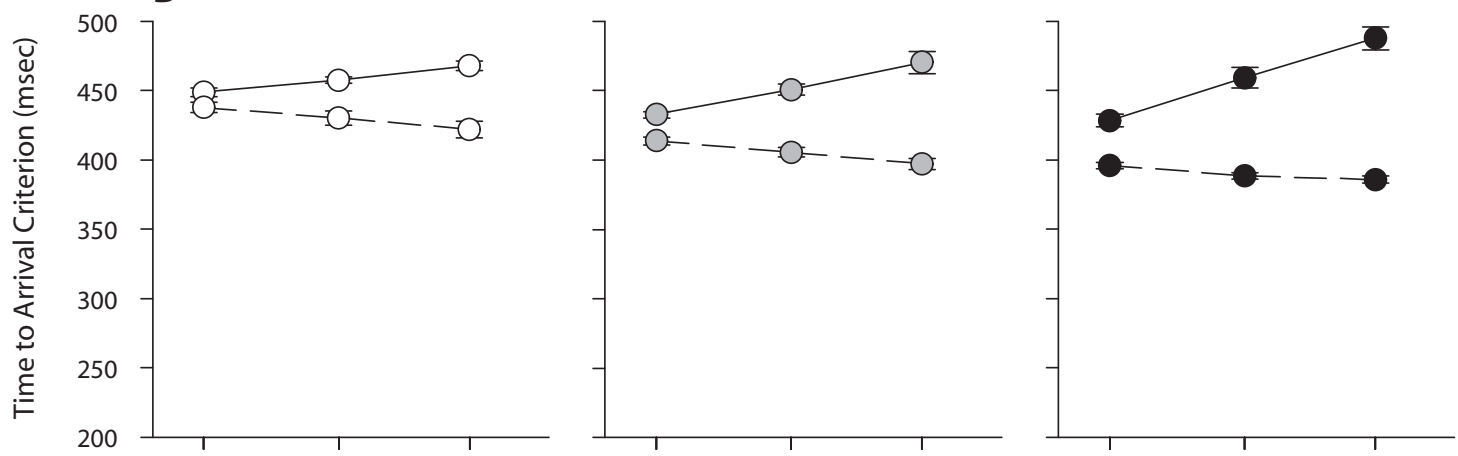

\section{C}
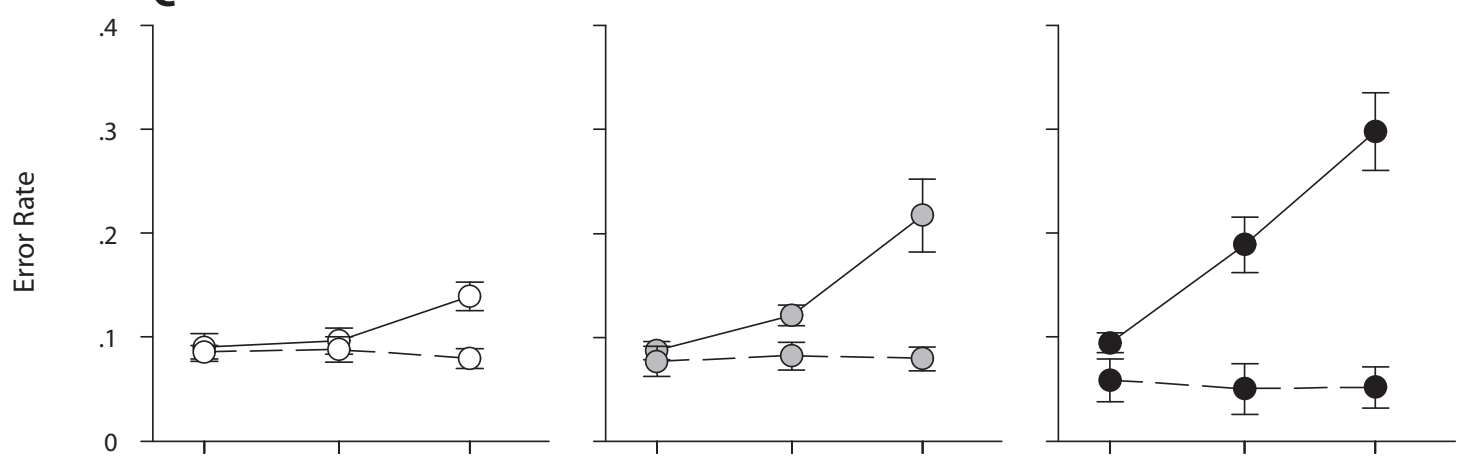

D
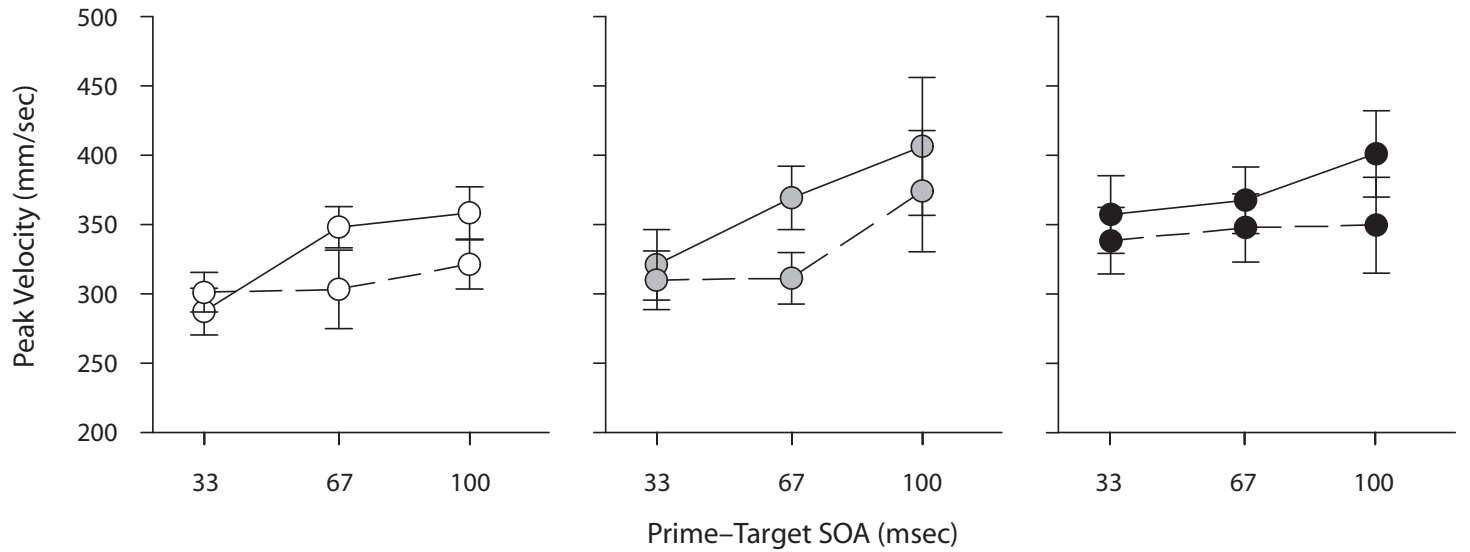

Figure 6. Parameters of the pointing trajectories. Details are as in Figure 3, except that the arrival criterion is on a radius $15 \mathrm{~mm}$ around the correct target. 


\section{Experiment 2: Parameters of Trajectorial Priming Functions}
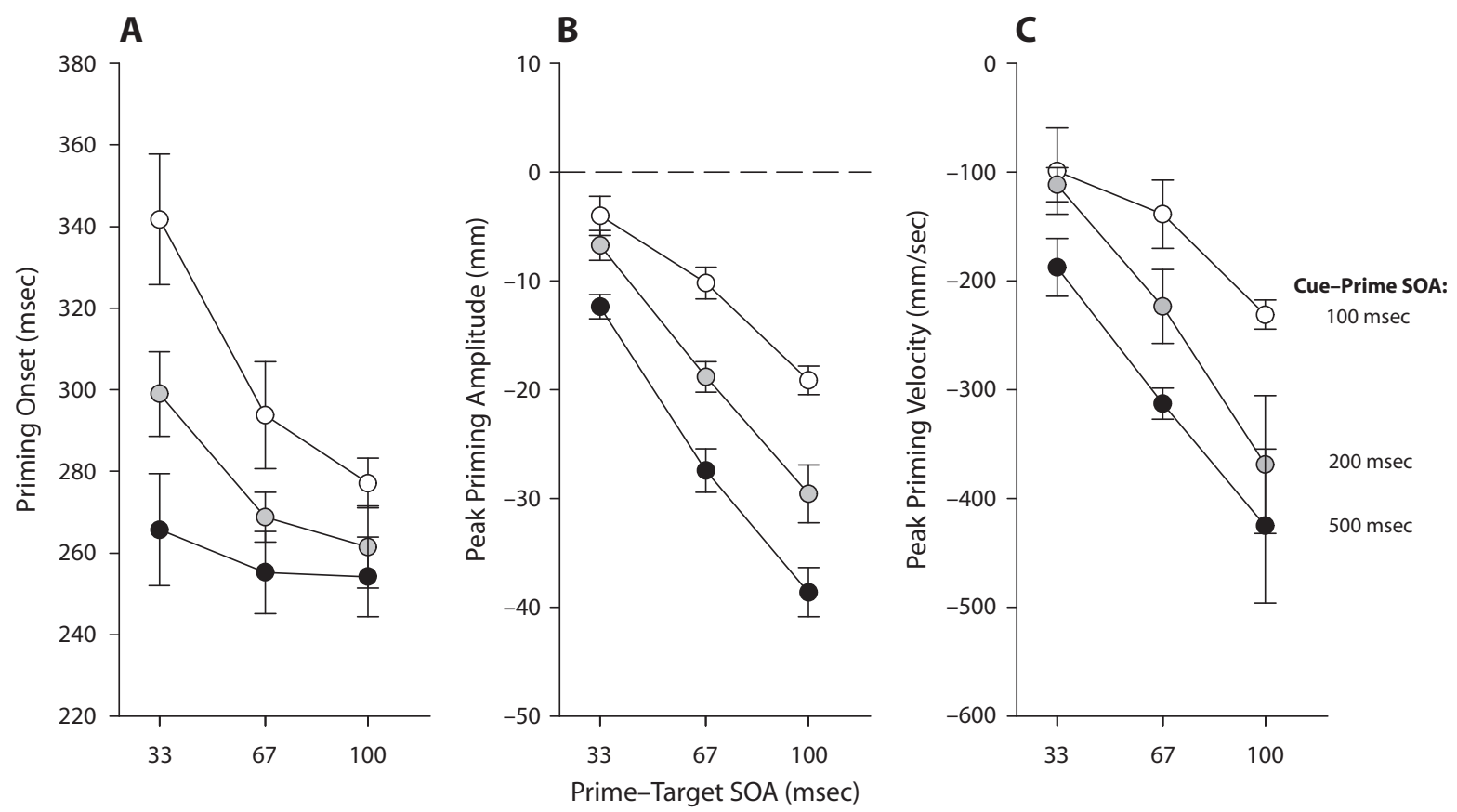

Figure 7. Parameters of trajectorial priming functions. Details are as in Figure 4.

However, priming effects at the 33-msec SOA start out too flatly in all conditions, reaching their onset criterion significantly too late (Figures $4 \mathrm{~A}$ and $7 \mathrm{~A}$ ); trajectories at this SOA show no signs of detours into the quadrant of the misleading prime, and thus fail on all three criteria. There might be several reasons for this departure from the rapidchase predictions. First, the rapid-chase criteria are defined for two sequential signals passing through the visuomotor system. Here, we have applied them to the second and third of three signals traversing the system, the first being the attentional cue. However, this does not explain why the rapid-chase predictions are met at the longer SOAs. Second, rapid chases have to break down at very short prime-target SOAs, because the temporal resolution of the system is finite: Ultimately, shorter and shorter SOAs must lead to overlap or mixing of the prime and target signals, attenuating the amplitude of the trajectorial priming function right from the start of the response. Up to now, however, we have observed breakdowns of the rapid-chase criteria only for SOAs as short as $17 \mathrm{msec}$ (Schmidt et al., 2006, Experiment 2), never at longer SOAs (Schmidt, 2002; Schmidt et al., 2006; Schmidt \& Schmidt, 2008; Vath \& Schmidt, 2007). All things considered, and with some caution applied, our results speak for rapid-chase processing of the primes and targets here, at least at the longer SOAs. This outcome supports the idea that the attentional modulation indeed affects the first, rapid waves of visuomotor activation (triggered sequentially by primes and targets) that traverse the visuomotor system.

Our general findings are in line with work by other authors. Modulation of response priming by temporal rather than spatial attention has been demonstrated by Naccache, Blandin, and Dehaene (2002) by using Dehaene et al.'s (1998) number-priming paradigm. In that study, priming effects occurred only when the onset time of the target was predictable, not when it was embedded in a context of unpredictable onset times, even if the prime-target SOA was preserved. Attentional modulation of semantic priming has been reported by Kiefer and Brendel (2006) and Lachter, Forster, and Ruthruff (2004) with exogenous as well as endogenous spatial cues.

Sumner, Tsai, Yu, and Nachev (2006) studied the effects of exogenous spatial cues on response priming of keypress responses. They presented a sequence of a prime arrow pointing either left or right, a pattern mask, and a target arrow. The primes and targets appeared independently either above or below the fixation point, and attention toward the prime was manipulated by flashing a visual precue $100 \mathrm{msec}$ before prime onset at either the prime's location or the opposite one. Consistent with our findings, the magnitude of the priming effect was larger for primes at cued than at uncued positions. Because the prime-target SOAs employed by Sumner et al. were much longer than those in the present study, in a range at which priming effects can actually reverse under suitable stimulus conditions (Eimer \& Schlaghecken, 1998, 2003; Lingnau \& Vorberg, 2005; Lleras \& Enns, 2004; Schlaghecken \& Eimer, 2002; Verleger et al., 2004), that study does not fully address the issue of how visual attention affects the earliest visuomotor signals from primes and targets that reach the level of response control.

Nevertheless, Sumner et al.'s (2006) study poses another interesting question. The authors tried to determine 
whether attentional enhancement reflected a modification of the priming process itself (motor enhancement - e.g., by increasing the gain of the motor response or lowering response thresholds) or an amplification of the prime's signal strength (signal enhancement - e.g., by increasing effective signal contrast or signal-to-noise ratio). Sumner et al. suggested opposite predictions for what the consequences of different types of enhancement would be when signal strength was directly varied. A test varying prime duration favored the motor enhancement account but confounded prime duration with prime-target SOA, and the results of a second test, which eliminated this confound by varying the prime's luminance contrast instead of duration, were consistent with the signal as well as the motor enhancement predictions. Sumner et al. concluded that these results could not be explained by signal enhancement alone, but implied some form of motor enhancement as well. However, their predictions regarding the effects of prime duration and intensity on the sign and amplitude of the priming effect are difficult to evaluate. In light of possible nonlinearities, such as mutual inhibition between responses (Vorberg et al., 2003) or even self-inhibition within responses (Schlaghecken \& Eimer, 2002), it is difficult to predict the effects of pure signal or motor enhancement without explicitly modeling the full time course of priming (Lingnau \& Vorberg, 2005).

The attentional effects reported here are in line with a signal enhancement interpretation: They are indistinguishable from those obtained by manipulating color contrast directly (Schmidt et al., 2006) and are consistent with the idea that visual attention enhances the effective contrast of the prime signal (Boynton, 2005; McAdams \& Maunsell, 1999; Reynolds \& Chelazzi, 2004; Reynolds et al., 2000; Treue \& Martínez Trujillo, 1999). Moreover, we can rule out at least two alternative interpretations. First, the increase in pointing velocities in valid as compared with invalid trials speaks against the notion that attention merely brings forward the time of processing of the prime without changing the subsequent time course of that processing. Second, Experiment 2 rules out the idea that attention has an unspecific (nondirectional) effect on the motor system - for example, by simply lowering the threshold for any upcoming motor response - because our task required spatial selection of the relevant pair of target locations as a prerequisite for performing the correct response at all.

However, we cannot rule out a third alternative: Attention might play a role in preactivating responses to both cued locations while concurrently suppressing preparation of responses to uncued locations (Rizzolatti, Riggio, Dascola, \& Umiltà, 1987; see also Mathews, Ainsley Dean, \& Sterr, 2006; Thompson, Biscoe, \& Sato, 2005). However, given the large number of demonstrations of attentional enhancement in early visual areas, it seems unlikely that motor enhancement alone could be responsible for our results, even though it might well complement any effects of signal enhancement.

\section{AUTHOR NOTE}

This work was supported by Deutsche Forschungsgemeinschaft Grant $1671 / 1$ to T.S. Correspondence may be sent to either author at the
University of Giessen, Department of General and Experimental Psychology, Otto-Behaghel-Str. 10F, D-35394 Giessen, Germany (e-mail: thomas.schmidt@psychol.uni-giessen.de, anna.seydell@psychol .uni-giessen.de).

\section{REFERENCES}

Allport, A. (1989). Visual attention. In M. I. Posner (Ed.), Foundations of cognitive science (pp. 631-682). Cambridge, MA: MIT Press.

Boynton, G. M. (2005). Attention and visual perception. Current Opinion in Neurobiology, 15, 465-469.

Breitmeyer, B. G., \& ÖĞMEn, H. (2006). Visual masking: Time slices through conscious and unconscious vision. Oxford: Oxford University Press.

Bullier, J. (2001). Integrated model of visual processing. Brain Research Reviews, 36, 96-107.

Carrasco, M., Penpeci-Talgar, C., \& Eckstein, M. (2000). Spatial covert attention increases contrast sensitivity across the CSF: Support for signal enhancement. Vision Research, 40, 1203-1215.

Corbetta, M., Miezin, F. M., Dobmeyer, S., Shulman, G. L., \& Petersen, S. E. (1990). Attentional modulation of neural processing of shape, color, and velocity in humans. Science, 248, 1556-1559.

Dehaene, S., Naccache, L., Le Clec'H, G., Koechlin, E., MuelLer, M., Dehaene-Lambertz, G., ET AL. (1998). Imaging unconscious semantic priming. Nature, 395, 597-600.

Desimone, R., \& Duncan, J. (1995). Neural mechanisms of selective visual attention. Annual Review of Neuroscience, 18, 193-222.

Di Lollo, V., Enns, J. T., \& Rensink, R. A. (2000). Competition for consciousness among visual events: The psychophysics of reentrant visual processes. Journal of Experimental Psychology: General, 129, 481-507.

Eimer, M., \& Schlaghecken, F. (1998). Effects of masked stimuli on motor activation: Behavioral and electrophysiological evidence. Journal of Experimental Psychology: Human Perception \& Performance, 24, 1737-1747.

Eimer, M., \& Schlaghecken, F. (2002). Links between conscious awareness and response inhibition: Evidence from masked priming. Psychonomic Bulletin \& Review, 9, 514-520.

Eimer, M., \& SCHLAGHeCKen, F. (2003). Response facilitation and inhibition in subliminal priming. Biological Psychology, 64, 7-26.

FranCIS, G. (1997). Cortical dynamics of lateral inhibition: Metacontrast masking. Psychological Review, 104, 572-594.

Kastner, S., \& Ungerleider, L. G. (2000). Mechanisms of visual attention in the human cortex. Annual Review of Neuroscience, 23, 315-341.

Kiefer, M., \& Brendel, D. (2006). Attentional modulation of unconscious "automatic" processes: Evidence from event-related potentials in a masked priming paradigm. Journal of Cognitive Neuroscience, 18, 184-198.

Klotz, W., \& Neumann, O. (1999). Motor activation without conscious discrimination in metacontrast masking. Journal of Experimental Psychology: Human Perception \& Performance, 25, 976-992.

Kunde, W., Kiesel, A., \& Hoffmann, J. (2003). Conscious control over the content of unconscious cognition. Cognition, 88, 223-242.

Lachter, J., Forster, K. I., \& Ruthruff, E. (2004). Forty-five years after Broadbent (1958): Still no identification without attention. Psychological Review, 111, 880-913.

LAMme, V. A. F. (2000). Neural mechanisms of visual awareness: A linking proposition. Brain \& Mind, 1, 385-406.

LAmme, V. A. F. (2006). Towards a true neural stance on consciousness. Trends in Cognitive Sciences, 10, 494-501.

Lamme, V. A. F., \& Roelfsema, P. R. (2000). The distinct modes of vision offered by feedforward and recurrent processing. Trends in Neurosciences, 23, 571-579.

Lamme, V. A. F., Zipser, K., \& Spekreisse, H. (2002). Masking interrupts figure-ground signals in V1. Journal of Cognitive Neuroscience, 14, 1044-1053.

Leuthold, H., \& Kopp, B. (1998). Mechanisms of priming by masked stimuli: Inferences from event-related brain potentials. Psychological Science, 9, 263-269.

Lingnau, A., \& Vorberg, D. (2005). The time course of response inhibition in masked priming. Perception \& Psychophysics, 67, 545-557.

LlerAs, A., \& ENNS, J. T. (2004). Negative compatibility or object up- 
dating? A cautionary tale of mask-dependent priming. Journal of Experimental Psychology: General, 133, 475-493.

Loftus, G. R., \& Masson, M. E. J. (1994). Using confidence intervals in within-subject designs. Psychonomic Bulletin \& Review, $\mathbf{1}$, 476-490.

Mathews, S., Ainsley Dean, P. J., \& Sterr, A. (2006). EEG dipole analysis of motor-priming foreperiod activity reveals separate sources for motor and spatial attention components. Clinical Neurophysiology, 117, 2675-2683.

Mattler, U. (2003). Priming of mental operations by masked stimuli. Perception \& Psychophysics, 65, 167-187.

McAdams, C. J., \& Maunsell, J. H. R. (1999). Effects of attention on orientation-tuning functions of single neurons in macaque cortical area V4. Journal of Neuroscience, 19, 431-441.

McMains, S. A., \& Somers, D. C. (2004). Multiple spotlights of attentional selection in human visual cortex. Neuron, 42, 677-686.

Naccache, L., Blandin, E., \& Dehaene, S. (2002). Unconscious masked priming depends on temporal attention. Psychological Science, 13, 416-424.

Neumann, O. (1990). Direct parameter specification and the concept of perception. Psychological Research, 52, 207-215.

Neumann, O., \& Klotz, W. (1994). Motor responses to nonreportable, masked stimuli: Where is the limit of direct parameter specification? In C. Umiltà \& M. Moscovitch (Eds.), Attention and performance $X V$ : Conscious and nonconscious information processing (pp. 123-150). Cambridge, MA: MIT Press, Bradford Books.

Reynolds, J. H., \& Chelazzi, L. (2004). Attentional modulation of visual processing. Annual Review of Neuroscience, 27, 611-647.

Reynolds, J. H., Pasternak, T., \& Desimone, R. (2000). Attention increases sensitivity of V4 neurons. Neuron, 26, 703-714.

Rizzolatti, G., Riggio, L., Dascola, I., \& Umiltà, C. (1987). Reorienting attention across the horizontal and vertical meridians: Evidence in favor of a premotor theory of attention. Neuropsychologia, 25, 31-40.

Schlaghecken, F., \& Eimer, M. (2002). Motor activation with and without inhibition: Evidence for a threshold mechanism in motor control. Perception \& Psychophysics, 64, 148-162.

Schmidt, T. (2002). The finger in flight: Real-time motor control by visually masked color stimuli. Psychological Science, 13, 112-118.

Schmidt, T., Niehaus, S., \& Nagel, A. (2006). Primes and targets in rapid chases: Tracing sequential waves of motor activation. Behavioral Neuroscience, 120, 1005-1016.

Schmidt, T., \& Schmidt, F. (2008). Processing of natural images is feedforward: A simple behavioral test. Manuscript submitted for publication.

Schmidt, T., \& Vorberg, D. (2006). Criteria for unconscious cognition: Three types of dissociation. Perception \& Psychophysics, 68, 489-504.

Sumner, P., Tsai, P.-C., Yu, K., \& Nachev, P. (2006). Attentional modulation of sensorimotor processes in the absence of perceptual awareness. Proceedings of the National Academy of Sciences, 103, 10520-10525.

Thompson, K. G., Biscoe, K. L., \& Sato, T. R. (2005). Neuronal basis of covert spatial attention in the frontal eye field. Journal of Neuroscience, 25, 9479-9487.
Thorpe, S., Fize, D., \& Marlot, C. (1996). Speed of processing in the human visual system. Nature, 381, 520-522.

Tong, F. (2003). Primary visual cortex and visual awareness. Nature Reviews Neuroscience, 4, 219-229.

Treue, S., \& Martínez Trujillo, J. C. (1999). Feature-based attention influences motion processing gain in macaque visual cortex. Nature, 399, 575-579.

Ulrich, R., \& Miller, J. (2001). Using the jackknife-based scoring method for measuring LRP onset effects in factorial designs. Psychophysiology, 38, 816-827.

VanRullen, R., \& КосH, C. (2003). Visual selective behavior can be triggered by a feed-forward process. Journal of Cognitive Neuroscience, 15, 209-217.

VANRullen, R., \& Thorpe, S. J. (2002). Surfing a spike wave down the ventral stream. Vision Research, 42, 2593-2615.

VAth, N., \& Schmidt, T. (2007). Tracing sequential waves of rapid visuomotor activation in lateralized readiness potentials. Neuroscience, 145, 197-208.

Verleger, R., Jaśkowski, P., Aydemir, A., van der Lubbe, R. H. J., \& GROEN, M. (2004). Qualitative differences between conscious and nonconscious processing? On inverse priming induced by masked arrows. Journal of Experimental Psychology: General, 133, 494-515.

Vorberg, D., Mattler, U., Heinecke, A., Schmidt, T., \& SchwarzBACH, J. (2003). Different time courses for visual perception and action priming. Proceedings of the National Academy of Sciences, 100, 6275-6280.

Yantis, S., \& Jonides, J. (1990). Abrupt visual onsets and selective attention: Voluntary versus automatic allocation. Journal of Experimental Psychology: Human Perception \& Performance, 16, 121-134.

Yantis, S., \& SEREnces, J. T. (2003). Cortical mechanisms of spacebased and object-based attentional control. Current Opinion in Neurobiology, 13, 187-193.

\section{NOTES}

1. In the context of neuronal signal flow, feedforward indicates that a cell passes activation on to another cell before integrating any feedback from other cells about that signal (VanRullen \& Koch, 2003).

2. In pointing responses, overt responses in the wrong direction occur mainly in trials with an early movement onset; in the majority of trials, priming is manifest in the delayed onset of responses (Schmidt et al., 2006). These covert processes can be examined electrophysiologically (Vath \& Schmidt, 2007). Here, we assume that the sequential response activation processes at work are identical for overt and covert priming effects.

3. Note that in many of our inconsistent conditions, this criterion could only be reached after any detour in a negative direction. Therefore, it is not strictly a criterion of movement onset. It was not possible to properly calculate onset times in a negative direction because detours were not always large enough to yield such a measure.

(Manuscript received January 25, 2007; revision accepted for publication October 10, 2007.) 